\title{
HEPATOMETRIA ULTRA-SONOGRÁFICA EM CRIANÇAS: PROPOSTA DE NOVO MÉTODO*
}

\author{
Silvia Maria Sucena da Rocha ${ }^{1}$, Ilka Regina Souza de Oliveira ${ }^{2}$, Azzo Widman ${ }^{3}$, Bety \\ Spilberg Karpovas Chisman ${ }^{4}$, Júlia Tizue Fukushima ${ }^{5}$, Luiz Antonio Nunes de Oliveira ${ }^{6}$, \\ Giovanni Guido Cerri ${ }^{7}$
}

Resumo OBJETIVO: Padronizar método ultra-sonográfico de biometria hepática em crianças, visando estabelecer maior precisão nos planos de corte e minimizar o fator operador-dependência; testar a reprodutibilidade intra-observador. CASUÍSTICA E MÉTODO: A hepatometria ultra-sonográfica foi realizada em 32 crianças, entre 0 e 6 anos de idade, sem doença hepática ou das vias biliares. Todas as crianças foram examinadas por um mesmo observador, em duas ocasiões diferentes (exames 1 e 2). Os planos seccionais foram estabelecidos interrelacionando linhas de orientação externas a reparos anatômicos intra-abdominais, extra e intra-hepáticos. Para análise comparativa das medidas dos exames 1 e 2 foi utilizado o teste t pareado. 0 coeficiente de Pearson foi empregado para análise de correlação: a) dos parâmetros entre si; b) entre a idade das crianças e a diferença das medidas dos exames 1 e 2. RESULTADOS: À análise estatística não houve: a) diferença significante no estudo da variabilidade intra-observador; b) correlação significante entre a diferença das medidas e as idades dos pacientes. Verificamos que os parâmetros estão, no geral, direta e altamente correlacionados entre si $(r>0,60)$. CONCLUSÃO: 0 método é reprodutível por um mesmo observador. As medidas dos diâmetros crânio-caudal na linha médio-esternal e crânio-caudal posterior na linha hemiclavicular, usando referenciais anatômicos intra-hepáticos, mostraram-se precisas e mais práticas que as demais. Unitermos: Fígado - dimensões; Biometria; Crianças; Ultra-som.

Abstract Sonographic biometry of the liver in children: proposal of a new method.

OBJECTIVE: The objectives of this study are: (1) to standardize the sonographic biometry method to determine the size of the liver in children in order to obtain a greater precision of the section planes and to minimize operator-dependant error; (2) to test intra-observer reproducibility. CASUISTIC AND METHOD: Sonographic measurements of the liver were performed by a single examiner in 32 children laged 0 to 6 years) with no liver or biliary system disease. The measurements were done in two different occasions (exams 1 and 2) to determine reproducibility. The section planes were based on external orientation lines related to intra-abdominal anatomic references, both extra and intra-hepatic. The values obtained in exams 1 and 2 were compared using a Student's paired t-test. The Pearson's coefficient was employed to determine the correlation among parameters as well as the correlation between the children's age and the difference in the values of exams 1 and 2. RESULTS: The statistical analysis revealed no significant intra-observer variability and no significant correlation between measurements' difference and children's age. In general, parameters were directly and highly correlated $(r>0.60)$. CONCLUSION: We concluded that the method is reproducible when performed by a same examiner. The measurements of the cranio-caudal diameter at the midsternal line and the posterior cranio-caudal diameter at the midclavicular line, based on intra-hepatic anatomic references, were precise and easier to perform than other routine measurements.

Key words: Liver size; Biometry; Children; Ultrasound.

* Trabalho realizado no Serviço de Diagnóstico por Imagem do Instituto da Criança (ICR) "Prof. Pedro de Alcântara" do Hospital das Clínicas da Faculdade de Medicina da Universidade de São Paulo (HC-FMUSP), São Paulo, SP.

1. Médica Colaboradora Voluntária do Serviço de Diagnóstico por Imagem do ICR/HC-FMUSP.

2. Doutora em Medicina, Diretora Técnica do Serviço de UItra-Sonografia do Instituto de Radiologia (InRad) do HC-FMUSP.

3. Doutor em Medicina, Médico Assistente Supervisor da Divisão de Cirurgia do Aparelho Digestivo II do HC-FMUSP.

4. Médica Radiologista Assistente do Serviço de Diagnóstico por Imagem do ICR/HC-FMUSP.

5. M.Sc., Estatística do Hospital Israelita Albert Einstein.

6. Chefe do Serviço de Diagnóstico por Imagem do ICR/HC FMUSP.

7. Professor Titular do Departamento de Radiologia da FMUSP, Chefe do InRad/HC-FMUSP.

Endereço para correspondência: Dra. Silvia Maria Sucena da Rocha. Serviço de Diagnóstico por Imagem, ICR/HC-FMUSP. Avenida Doutor Enéas de Carvalho Aguiar, 647, 2ª andar. São Paulo, SP, 05403-001. E-mail: smsucena@uol.com.br

Recebido para publicação em 1/10/2002. Aceito, após revisão, em 23/12/2002.

\section{INTRODUÇÃO}

A determinação do tamanho do fígado é procedimento de rotina no exame clínico de crianças, tanto para diagnóstico de hepatomegalia como para monitorar alterações das dimensões do órgão que possam se manifestar na evolução natural de doenças ou em consequiência a terapia. Para tanto, é necessária uma técnica de mensuração acurada e reprodutível, que defina valores normais de referência.

A hepatometria in vivo pode ser realizada pelo método clínico e por métodos de imagem: radiografia, cintilografia, ultra-sonografia e tomografia computadorizada.
Entre os autores é consenso que a projeção da borda hepática abaixo da borda costal, isoladamente, não constitui parâmetro confiável para a estimativa do tamanho do fígado ${ }^{(1-11)}$

Para melhor avaliação clínica, é proposto mensurar o comprimento hepático ("liver span") na linha hemiclavicular, ou seja, a distância entre as superfícies hepáticas superior e inferior, determinadas pelo método da percussão ${ }^{(6,12)}$ ou percussãopalpação $^{(\mathbf{4}, 5,7,13)}$.

A medida do comprimento hepático, obtida pelo método clínico, apresenta boa correlação com o tamanho do fígado, determinado por necropsia ${ }^{(\mathbf{1})}$. Na infância, o 
aumento do comprimento hepático é progressivo e tem boa correlação com a idade, peso, altura e superfície corpórea ${ }^{(5)}$.

A avaliação clínica, porém, apresenta limitações, decorrentes de variações na forma, eixo e posição do fígado ${ }^{(\mathbf{1 4}, 15)}$, de suas relações com estruturas vizinhas ${ }^{(6,15)}$ e da técnica de mensuração empregada ${ }^{(4,12)}$, sendo operador dependente ${ }^{(16)}$.

Em virtude disso, os métodos de diagnóstico por imagem passaram a ser utilizados, procurando correlacionar seus achados aos do método clínico e propondo novas formas de estudo do fígado que oferecessem subsídios mais concretos, com melhor reprodutibilidade, na tentativa de complementar a avaliação clínica. Esses métodos, ao propor dados quantitativos, procuram minimizar a variabilidade intra $\mathrm{e}$ interobservador.

Estudos de mensuração hepática têm sido realizados em adultos e crianças, utilizando-se radiografia ${ }^{(13,17-19)}$, cintilografia $^{(\mathbf{1 , 3 , 1 4 , 2 0 , 2 1})}$, tomografia computadoriza-

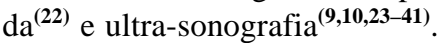

Dentre os métodos de imagem, a ultrasonografia apresenta vantagens, sobretudo na avaliação do paciente pediátrico, por se tratar de método totalmente não-invasivo, não utilizar radiação ionizante e não exigir sedação. Ademais, trata-se de método que permite o estudo minucioso do parênquima e estruturas hepáticas, bem como de suas relações com estruturas vizinhas. Por ser um método de custo relativamente baixo, de execução rápida, amplamente disponível e isento de riscos, a ultra-sonografia é, de modo geral, o primeiro exame de imagem solicitado para esclarecimento diagnóstico quando há suspeita clínica de hepatomegalia.

O método ultra-sonográfico, no entanto, tem algumas limitações, relevantes, no que se refere ao estudo do fígado. Essas limitações devem-se, em especial, à localização do fígado e traduzem-se, à ultra-sonografia, como uma dificuldade em definir seu limite superior, quer pela interposição de parênquima pulmonar entre o fígado e a parede torácica, quer pela presença dos arcos costais, pois o ar e os ossos constituem barreiras ao som.

A velocidade do som em tecidos que contêm ar é acentuadamente menor, em relação a outros tecidos, devido à alta com- placência do meio gasoso. Além disso, o ar promove atenuação do feixe sonoro, em decorrência da deflexão do som. Os ossos, por sua vez, promovem intensa absorção acústica e impedem a visualização das estruturas situadas abaixo deles. Para obter melhor definição das margens hepáticas, em adultos, recorre-se à parada da respiração em inspiração máxima. Em crianças pequenas não contamos com este recurso.

Na tentativa de escapar desses obstáculos ao som, podem ser tentadas angulações do transdutor. No entanto, pequenas mudanças no ângulo do transdutor fornecem imagens bastante diferentes e, por conseguinte, medidas também diferentes.

A forma do fígado constitui outro fator de dificuldade para a padronização de medidas, pois, sendo bizarra, grosseiramente semelhante a uma cunha e com ampla superfície superior, arredondada, exige definição de pontos de referência precisos quando se pretende padronizar uma técnica com boa reprodutibilidade.

A literatura é relativamente pobre no que se refere às medidas do fígado. Mesmo os livros-texto não dedicam muita importância ao tema e, quando o fazem, em geral, não vão além de descrever pontos de referência básicos para as medidas. As convenções para efetuar essas medidas, por falta de indicações precisas, permitem diferentes interpretações de como tomá-las. Podem ser obtidas medidas diversas num mesmo plano, bastando para tal a escolha de pontos diferentes a serem atingidos na borda hepática superior.

Os trabalhos que tratam da hepatometria em crianças por meio da ultra-sonogra-

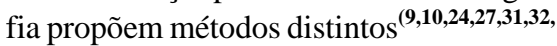
36-41), alguns não passíveis de reprodução ${ }^{(\mathbf{2 4 , 2 7 , 3 1 )}}$, já que os aparelhos utilizados então (aparelhos estáticos) tornaram-se obsoletos, tendo sido substituídos pelos aparelhos de rastreamento em tempo real. A nosso ver, a multiplicidade de métodos de mensuração do fígado reflete a falta de um que atenda às necessidades do examinador, ou seja, uma técnica confiável, reprodutível e de simples execução.

O presente estudo tem por objetivo estabelecer e padronizar pontos de referência para a obtenção das medidas do fígado de crianças, avaliando sua reprodutibilidade intra-observador.

\section{CASUÍSTICA E MÉTODO}

\section{Casuística}

Entre março de 2001 e fevereiro de 2002 foram examinadas 32 crianças ( 16 do sexo feminino e 16 do sexo masculino), com idades entre 18 dias e 5 anos, 11 meses e 25 dias (média de 29,66 meses), provenientes da Enfermaria de Nefrologia do Instituto da Criança (ICR) "Prof. Pedro de Alcântara" do Hospital das Clínicas da Faculdade de Medicina da Universidade de São Paulo (HC-FMUSP) e da creche do HC-FMUSP.

Para o estudo da variabilidade intraobservador, cada uma das crianças foi examinada duas vezes pelo mesmo médico ultra-sonografista, com intervalo de tempo entre os exames de, no mínimo, 24 horas e, no máximo, 72 horas.

Todas as crianças apresentaram exames ultra-sonográficos normais. Foram excluídas da amostra crianças com doenças hepáticas e/ou das vias biliares.

\section{Método}

Os exames ultra-sonográficos foram realizados em modo-B, com equipamentos ATL modelo Apogee 800 Plus e Aloka modelo SS-2000, utilizando-se transdutor convexo de 5,0 MHz.

\section{1 - Técnica de exame}

As crianças foram examinadas na posição supina, com membros superiores e inferiores estendidos ao lado do corpo, a cabeça apoiada sobre travesseiro baixo, não se fazendo uso de preparo ou sedação.

$\mathrm{O}$ transdutor foi posicionado abaixo do gradeado costal, com orientação longitudinal, cuidando-se para que estivesse sempre em posição ortogonal em relação ao plano da coluna. Para a identificação da interface visceral e diafragmática do lobo direito foi necessária leve angulação do transdutor no sentido cranial.

As imagens escolhidas para a realização das medidas foram aquelas que permitiram a melhor definição dos limites do órgão, não se levando em conta o instante respiratório do paciente.

Foi solicitado às crianças maiores, que colaboravam, que distendessem o abdome, quando essa manobra permitia maior precisão na aquisição da imagem. 


\section{2- Dinâmica do exame}

As medidas do fígado foram obtidas tomando-se como base linhas de orientação externas, correlacionadas a reparos anatômicos intra e extra-hepáticos.

O plano de corte do lobo esquerdo foi estabelecido por uma linha médio-esternal (LME), traçada do ponto médio do esterno à cicatriz umbilical, com identificação simultânea da veia hepática esquerda, excluindo-se da imagem a veia cava inferior.

Os planos de corte do lobo hepático direito foram estabelecidos por: a) linha hemiclavicular (LHC), traçada desde o ponto médio da clavícula até o ponto médio da prega inguinal, com simultânea iden- tificação do ramo portal direito, em secção transversal e ampla visualização do diafragma; b) linha axilar anterior (LAA), traçada verticalmente a partir da prega axilar anterior, tomando-se como referência intrahepática o ramo portal direito e como referências extra-hepáticas o rim direito, o músculo psoas e ampla visualização do diafragma (Figura 1).

\section{3 - Medidas}

Nos planos de corte, foram feitas as seguintes medidas:

\section{Lobo hepático esquerdo}

Na LME: a) medida do diâmetro crâniocaudal (CC-LME), através de uma linha horizontal, paralela à parede abdominal, estendendo-se desde a superfície diafragmática até a borda hepática inferior; b) medida do diâmetro ântero-posterior (APLME), através de uma linha vertical, perpendicular ao diâmetro crânio-caudal, unindo as extremidades superiores anterior e posterior do lobo, até o ligamento venoso (Figura 2).

\section{Lobo hepático direito}

Na LHC: c) medida do diâmetro crâniocaudal da porção anterior do fígado (CCALHC), através de uma linha horizontal, paralela à parede abdominal, estendendose da superfície diafragmática até a borda inferior do lobo; d) medida do diâmetro
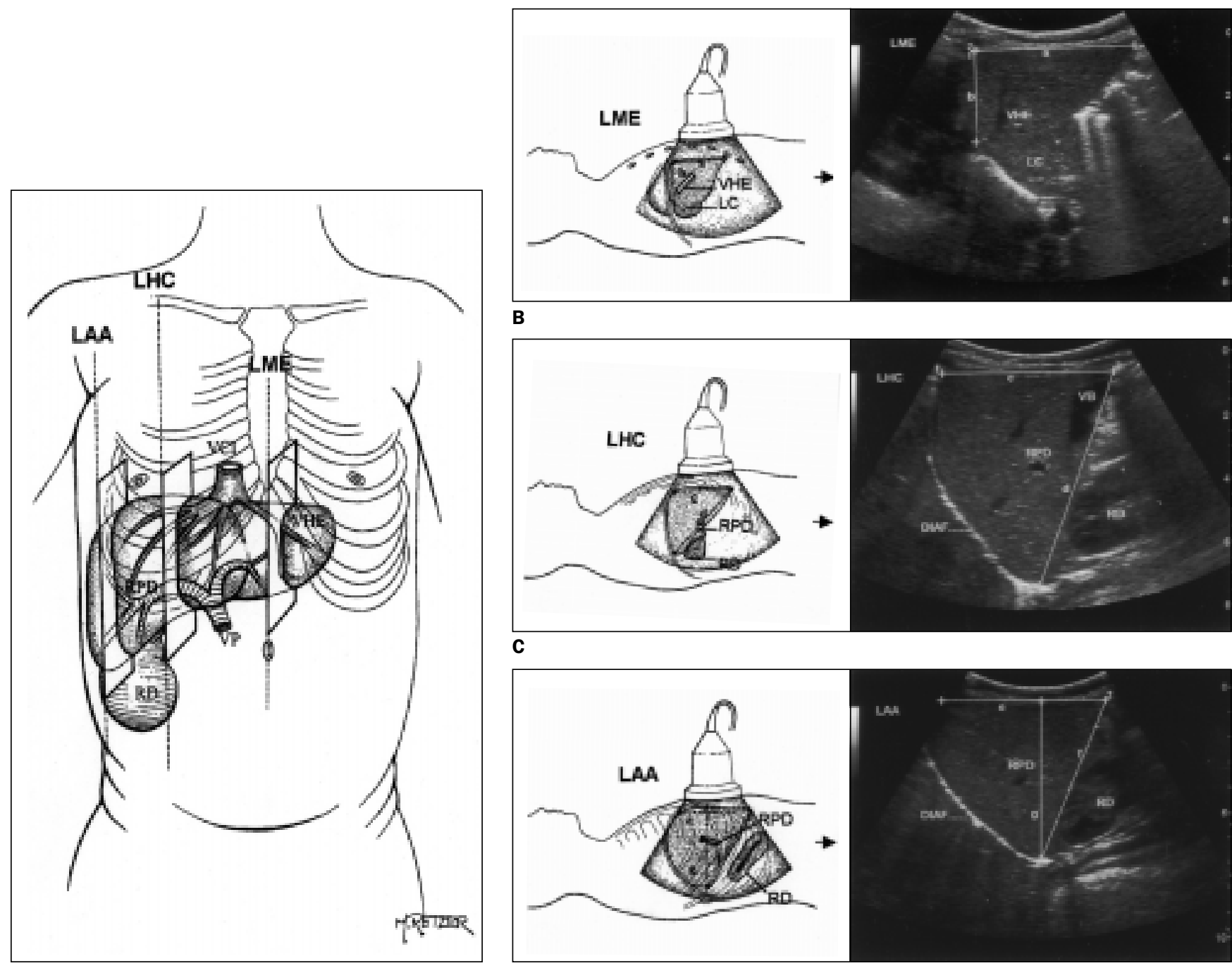

B

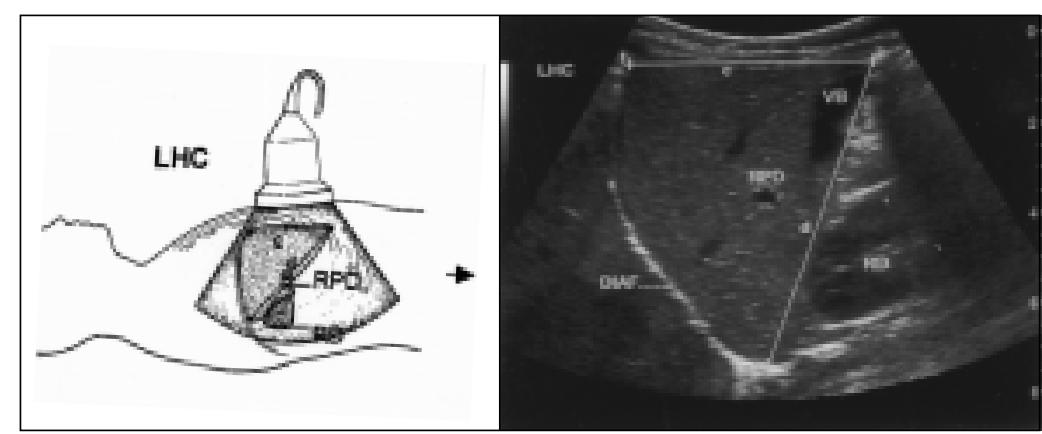

C

A

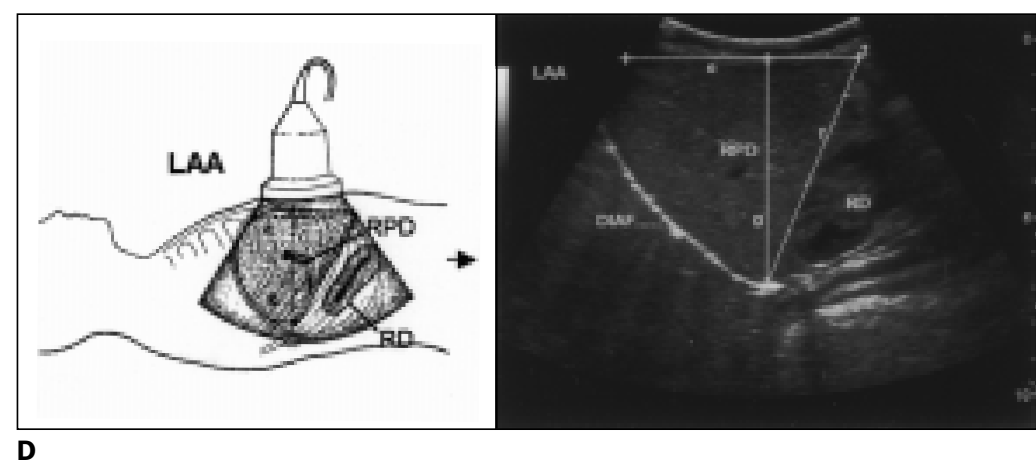

Figura 1. Representação esquemática e documentação fotográfica do exame ultra-sonográfico, mostrando planos de corte, pontos de referência e parâmetros para a determinação das dimensões hepáticas. LME, linha médio-esternal; LHC, linha hemiclavicular; LAA, linha axilar anterior; VCl, veia cava inferior; VHE, veia hepática esquerda; VP, veia porta; RPD, ramo portal direito; RD, rim direito; a, b, diâmetros crânio-caudal e ântero-posterior do lobo hepático esquerdo na linha médio-esternal; c,d, diâmetros crânio-caudal anterior e crânio-caudal posterior do lobo hepático direito na linha hemiclavicular; e, f, g, diâmetros crânio-caudal anterior, crânio-caudal posterior e ântero-posterior do lobo hepático direito na linha axilar anterior. 
crânio-caudal da porção posterior do fígado (CCP-LHC), através de uma linha oblíqua traçada entre a extremidade superior e a borda hepática inferior (Figura 3 ).

Na LAA: e) medida do diâmetro crâniocaudal da porção anterior do fígado (CCALAA), através de uma linha paralela à parede abdominal, traçada entre a superfície diafragmática e a borda hepática inferior; f) medida do diâmetro crânio-caudal da porção posterior do fígado (CCP-LAA), através de uma linha oblíqua, traçada entre a extremidade póstero-superior do lobo e sua borda inferior; g) medida do eixo ântero-posterior máximo (AP-LAA), através de uma linha vertical, perpendicular ao diâmetro crânio-caudal da porção anterior do fígado, traçada entre as superfícies anterior e posterior (Figura 4).

As medidas foram expressas em milímetros (mm), até duas casas decimais. Neste trabalho utilizamos o conceito de parâmetro como a medida realizada.

\section{Análise estatística}

Para a avaliação da reprodutibilidade intra-observador foram comparadas as médias de cada medida obtida nos dois exames de cada paciente e analisadas pelo teste t de "Student" pareado. Utilizamos o coeficiente de Pearson para avaliar: a) a correlação entre a idade das crianças e a variação das medidas dos exames 1 e 2 ; b) a correlação dos parâmetros entre si. Utilizou-se o nível de significância de 0,05.

\section{RESULTADOS}

\section{Variabilidade intra-observador}

Os resultados do estudo da variabilidade intra-observador mostraram não haver diferença significante entre as medidas afe- ridas nos exames 1 e 2 para qualquer dos parâmetros avaliados. Os dados são apresentados na Tabela 1.

\section{Correlação entre a variação das medidas e as idades das crianças}

A diferença entre os valores das medidas obtidas nos exames 1 e 2 não apresentou correlação estatisticamente significante com as idades das crianças (Tabela 2). Entretanto, observou-se que as diferenças referentes aos parâmetros AP-LME e CCPLHC tiveram correlação negativa com as idades, havendo maiores variações nas crianças com idades mais baixas.

\section{Análise de correlação entre os parâmetros}

A análise de correlação dos sete parâmetros avaliados demonstrou que todos estão diretamente correlacionados entre si,

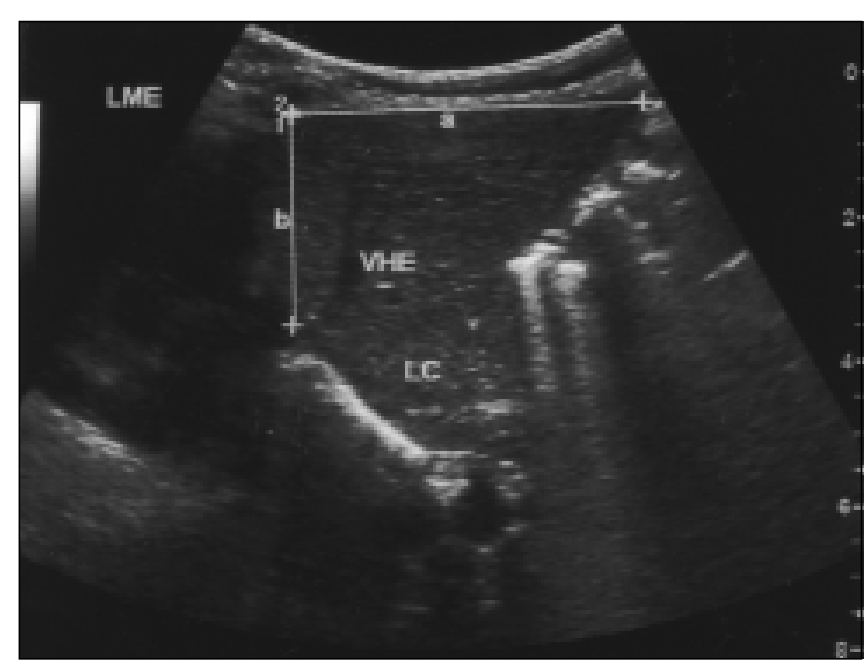

2

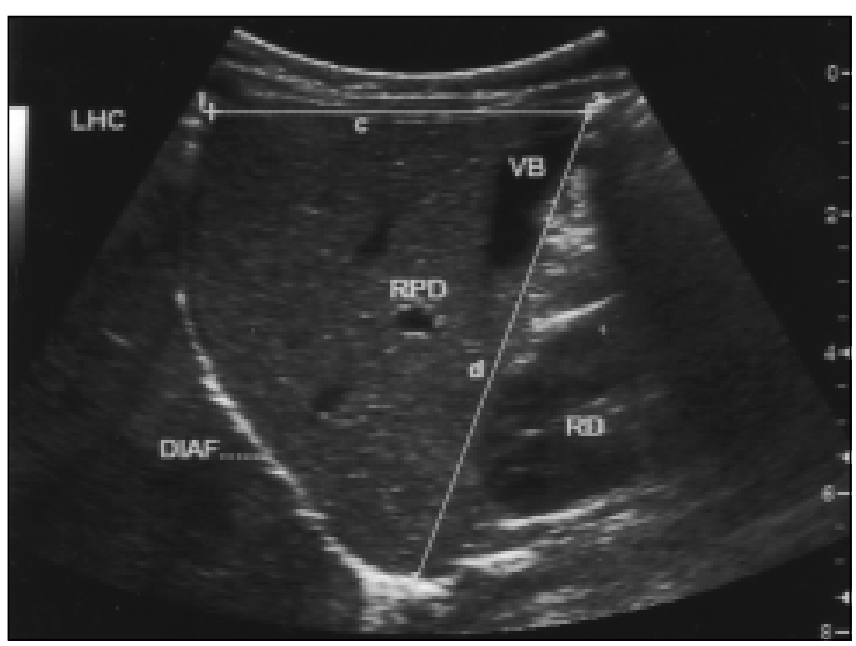

3
Figura 2. Lobo hepático esquerdo no plano longitudinal. LME, linha médio-esternal; VHE, veia hepática esquerda (reparo anatômico intra-hepático); LC, lobo caudado; a,diâmetro crânio-caudal; b, diâmetro ântero-posterior.

Figura 3. Lobo hepático direito no plano longitudinal. LHC, linha hemiclavicular; RPD, ramo portal direito (reparo anatômico intra-hepático); RD, rim direito; DIAF, diafragma (reparos anatômicos extra-hepáticos); VB, vesícula biliar: c, diâmetro crânio-caudal anterior; d, diâmetro crânio-caudal posterior.

Figura 4. Lobo hepático direito no plano longitudinal. LAA, linha axilar anterior; $\mathrm{RPD}$, ramo portal direito (reparo anatômico intra-hepático); RD, rim direito; DIAF, diafragma (reparo anatômico extra-hepático); e, diâmetro crânio-caudal anterior; f, diâmetro crânio-caudal posterior; g, diâmetro ântero-posterior.

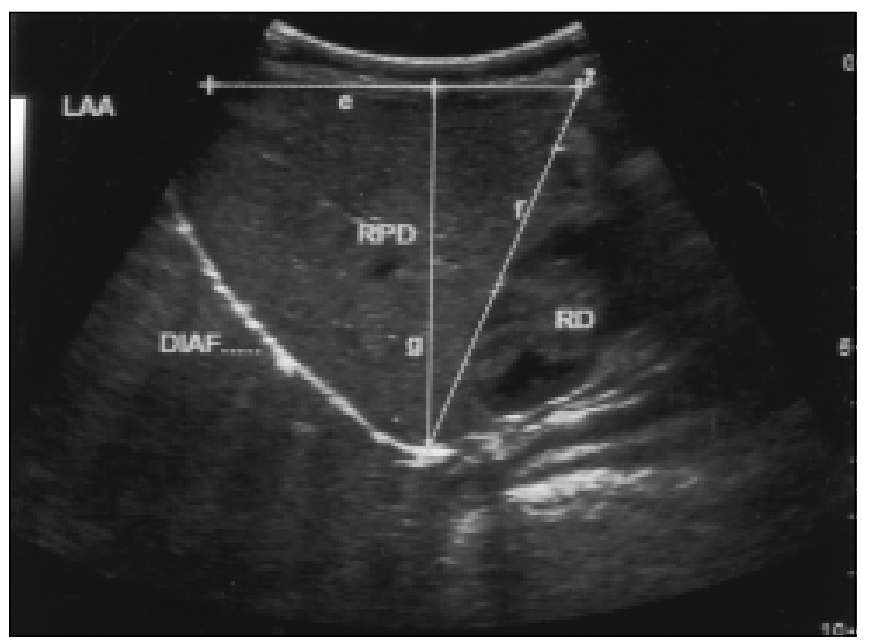

4 
exceto AP-LME em relação a CCP-LAA (Tabela 3).

CCA-LHC e CCP-LHC apresentaram altos coeficientes de correlação $(>0,70)$ em relação a cinco parâmetros, só tendo baixa correlação com AP-LME.

CC-LME, CCA-LAA e AP-LAA apresentaram altos coeficientes de correlação $(>0,70)$ em relação a quatro parâmetros.
AP-LME apresentou baixos coeficientes de correlação, todos menores que 0,60.

\section{DISCUSSÃO}

O aumento do fígado, decorrente de doença hepática intrínseca ou afecções sistêmicas, é um achado clínico comum na infância $^{(11)}$. Grandes aumentos do órgão são

Tabela 1 Médias das medidas do fígado obtidas nos exames 1 e 2. Desvio-padrão (DP) e valores do teste t pareado $(p)$.

\begin{tabular}{|l|c|c|c|}
\hline & $\begin{array}{c}\text { Exame 1 } \\
\text { Média } \pm \mathrm{DP}(\mathrm{mm})\end{array}$ & $\begin{array}{c}\text { Exame 2 } \\
\text { Média } \pm \mathrm{DP}(\mathrm{mm})\end{array}$ & $\begin{array}{c}(\mathrm{n}=32) \\
\mathrm{p}\end{array}$ \\
\hline CC-LME & $58,12 \pm 11,83$ & $59,82 \pm 12,08$ & 0,163488 \\
AP-LME & $42,95 \pm 6,06$ & $43,41 \pm 6,19$ & 0,585347 \\
CCA-LHC & $57,11 \pm 9,05$ & $55,97 \pm 10,33$ & 0,380407 \\
CCP-LHC & $90,63 \pm 12,40$ & $91,23 \pm 11,20$ & 0,621326 \\
CCA-LAA & $71,95 \pm 11,30$ & $72,96 \pm 10,47$ & 0,419654 \\
CCP-LAA & $92,66 \pm 12,71$ & $93,67 \pm 11,35$ & 0,473524 \\
AP-LAA & $75,87 \pm 9,89$ & $74,04 \pm 9,02$ & 0,15552 \\
\hline
\end{tabular}

CC-LME, eixo crânio-caudal na linha médio-esternal; AP-LME, eixo ântero-posterior na linha médio-esternal; CCALHC, eixo crânio-caudal anterior na linha hemiclavicular; CCP-LHC, eixo crânio-caudal posterior na linha hemiclavicular; CCA-LAA, eixo crânio-caudal anterior na linha axilar anterior; CCP-LAA, eixo crânio-caudal posterior na linha axilar anterior; AP-LAA, eixo ântero-posterior na linha axilar anterior.

Tabela 2 Coeficiente de correlação de Pearson e probabilidade de significância entre a diferença das médias dos parâmetros e as idades.

\begin{tabular}{|l|l|l|l|l|l|l|l|}
\hline \multirow{4}{*}{ Idade } & CC-LME & AP-LME & CCA-LHC & CCP-LHC & CCA-LAA & CCP-LAA & AP-LAA \\
& 0,07052 & $-0,26547$ & 0,00887 & $-0,27332$ & 0,06401 & 0,14429 & 0,02946 \\
& $p=0,7013$ & $p=0,1420$ & $p=0,9616$ & $p=0,1301$ & $p=0,7378$ & $p=0,4308$ & $p=0,6184$ \\
\hline
\end{tabular}

CC-LME, eixo crânio-caudal na linha médio-esternal; AP-LME, eixo ântero-posterior na linha médio-esternal; CCALHC, eixo crânio-caudal anterior na linha hemiclavicular; CCP-LHC, eixo crânio-caudal posterior na linha hemiclavicular; CCA-LAA, eixo crânio-caudal anterior na linha axilar anterior; CCP-LAA, eixo crânio-caudal posterior na linha axilar anterior; AP-LAA, eixo ântero-posterior na linha axilar anterior.

$p<0,05$, significante.

facilmente identificados pelo exame clínico, no entanto, frente à suspeita clínica de um pequeno aumento hepático, são solicitados exames subsidiários que permitam elucidar a dúvida diagnóstica. A ultra-sonografia é o método de escolha para dar prosseguimento à investigação diagnóstica, dadas suas características de exame totalmente não invasivo, largamente disponível e de fácil execução.

Vários métodos ultra-sonográficos de biometria hepática têm sido descritos. Os primeiros trabalhos, realizados em aparelhos estáticos, descreviam, em sua maioria, métodos de estimativa do volume e área hepática através de múltiplos planos de

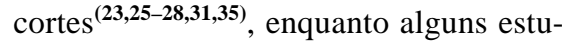
dos propunham a validade de se avaliar as dimensões do órgão com base em apenas um plano de corte longitudinal ${ }^{(\mathbf{2 4 , 3 0 )}}$. Com o advento dos aparelhos de ultra-sonografia em tempo real, caíram em desuso os aparelhos estáticos e, com eles, os métodos mais complexos, passando a ser aceitos, então, aqueles baseados em poucos planos de corte longitudinais ${ }^{(32,33)}$, que se tornaram referência para os trabalhos ul-

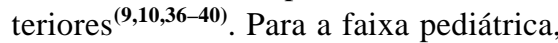
inicialmente foi estabelecido normograma ultra-sonográfico a partir de método que correlaciona linhas de orientação externas a reparos anatômicos intra-abdominais, extra-hepáticos ${ }^{(32)}$. Com a finalidade de aprimorar esse método, no presente estudo, além de inter-relacionarmos linhas de

Tabela 3 Coeficientes de correlação de Pearson e probabilidade de significância na comparação das médias dos parâmetros entre si.

\begin{tabular}{|l|lllllll|}
\hline & CC-LME & AP-LME & CCA-LHC & CCP-LHC & CCA-LAA & CCP-LAA & AP-LAA \\
\hline CC-LME & 1 & 0,4908 & $\mathbf{0 , 8 2 9 7}$ & $\mathbf{0 , 7 9 0 8}$ & 0,5934 & $\mathbf{0 , 7 3 2 9}$ & $\mathbf{0 , 8 1 5 8}$ \\
& & $p=0,0043$ & $p<0,0001$ & $p<0,0001$ & $p=0,0003$ & $p<0,0001$ & $p<0,0001$ \\
AP-LME & 0,4908 & 1 & 0,4220 & 0,5739 & 0,5223 & 0,2607 & 0,4226 \\
& $p=0,0043$ & & $p=0,0161$ & $p=0,0006$ & $p=0,0022$ & $p=0,1496$ & $p=0,0160$ \\
CCA-LHC & $\mathbf{0 , 8 2 9 7}$ & 0,4220 & 1 & $\mathbf{0 , 7 5 7 6}$ & $\mathbf{0 , 7 7 2 6}$ & $\mathbf{0 , 7 5 8 2}$ & $\mathbf{0 , 8 1 0 4}$ \\
& $p<0,0001$ & $p=0,0161$ & & $p<0,0001$ & $p<0,0001$ & $p<0,0001$ & $p<0,0001$ \\
CCP-LHC & $\mathbf{0 , 7 9 0 8}$ & 0,5739 & $\mathbf{0 , 7 5 7 6}$ & 1 & $\mathbf{0 , 7 3 6 0}$ & $\mathbf{0 , 8 3 2 6}$ & $\mathbf{0 , 8 4 3 4}$ \\
& $p<0,0001$ & $p=0,0006$ & $p<0,0001$ & & $p<0,0001$ & $p<0,0001$ & $p<0,0001$ \\
CCA-LAA & 0,5934 & 0,5223 & $\mathbf{0 , 7 7 2 6}$ & $\mathbf{0 , 7 3 6 0}$ & 1 & 0,6107 & 0,5971 \\
& $p=0,0003$ & $p=0,0022$ & $p<0,0001$ & $p<0,0001$ & & $p=0,0002$ \\
CCP-LAA & $\mathbf{0 , 7 3 2 9}$ & 0,2607 & $\mathbf{0 , 7 5 8 2}$ & $\mathbf{0 , 8 3 2 6}$ & 0,6107 & 1 & $\mathbf{p}=0,0003$ \\
& $p<0,0001$ & $p=0,1496$ & $p<0,0001$ & $p<0,0001$ & $p=0,0002$ & $\mathbf{0 , 8 7 5 7}$ \\
AP-LAA & $\mathbf{0 , 8 1 5 8}$ & 0,4226 & $\mathbf{0 , 8 1 0 4}$ & $\mathbf{0 , 8 4 3 4}$ & 0,5971 & $\mathbf{0 , 8 7 5 7}$ \\
& $p<0,0001$ & $p=0,016$ & $p<0,0001$ & $p<0,0001$ & $p=0,0003$ & $p<0,0001$ \\
\hline
\end{tabular}

CC-LME, eixo crânio-caudal na linha médio-esternal; AP-LME, eixo ântero-posterior na linha médio-esternal; CCA-LHC, eixo crânio-caudal anterior na linha hemiclavicular; CCP-LHC, eixo crânio-caudal posterior na linha hemiclavicular; CCA-LAA, eixo crânio-caudal anterior na linha axilar anterior; CCP-LAA, eixo crânio-caudal posterior na linha axilar anterior; AP-LAA, eixo ântero-posterior na linha axilar anterior.

Valores em negrito: coeficientes de correlação $>0,70 . p<0,05$, significante. 
orientação externas a reparos anatômicos intra-abdominais extra-hepáticos, acrescentamos reparos anatômicos intra-hepáticos. Desse modo, visamos definir de modo mais preciso o plano de corte para aferição das medidas e, assim, minimizar o fator operador-dependência.

\section{Linha médio-esternal (Figura 2)}

As medidas do lobo hepático esquerdo na LME não apresentaram dificuldades técnicas relevantes, sendo possível, em todos os casos, definir adequadamente os limites do órgão e estabelecer os pontos de referência para as medidas dos diâmetros CC-LME e AP-LME. A inclusão da veia hepática esquerda como ponto de referência intra-hepática facilitou a determinação do local de medida no reexame, pois que a imagem do lobo hepático esquerdo pode assumir configurações diferentes com mínimas variações na posição do transdutor.

\section{Linha hemiclavicular (Figura 3)}

A LHC é referência padrão para a mensuração do fígado pelo método clínico, sendo reproduzida na maioria dos estudos de hepatometria pelos métodos de medicina nuclear e ultra-sonografia ${ }^{(1,4-7,12-14,16}$, ${ }^{20,41)}$. Sua utilização, porém, é controversa, tendo sido relatada substancial variação interobservador na estimativa da localização da LHC, passando a ser considerada um marco incerto ("wandering landmark") ${ }^{(\mathbf{4 2})}$. Entretanto, em nossa investigação constatamos que, quando associada ao reparo anatômico intra-hepático (ramo direito da veia porta próximo à bifurcação portal), a LHC deixa de ter caráter incerto, tornando-se ponto de referência consistente.

No plano obtido nesta linha medimos os diâmetros CCA-LHC e CCP-LHC e nos valemos de dois reparos anatômicos na superfície hepática superior: a interface hepato-diafragmática anterior e a interface hepato-diafragmática posterior, respectivamente.

\section{a) $C C A-L H C$}

O diâmetro CCA-LHC mede o comprimento da superfície anterior do fígado na LHC ("liver span") e é usualmente utilizado para avaliação das dimensões hepáticas pelo método clínico ${ }^{(4-7,12,14,16)}$. Tem sido reproduzido pela ultra-sonografia, servin- do como parâmetro, tanto na confecção de normogramas como em estudos comparativos com outros métodos ${ }^{(\mathbf{9}, 10,32,33,36-39)}$.

Um dos reparos anatômicos para a determinação do CCA-LHC é a interface hepato-diafragmática anterior, que pode apresentar-se imprecisa, devido à interposição de arcos costais e parênquima pulmonar entre o transdutor e o fígado. Em nossa casuística não foi possível a identificação dessa interface em dois pacientes $(6,25 \%)$, tendo-se tomado, então, como referência, a interface hepato-pulmonar ${ }^{(24,33)}$. No entanto, a seleção do ponto limite dessa interface pode resultar em discrepância das medidas $^{(\mathbf{2 4})}$. Em estudo ultra-sonográfico de hepatometria em crianças foi observado que a imprecisão da interface hepatodiafragmática anterior teria se dado em conseqüência da utilização de transdutor linear, não ocorrendo com o uso de transdutor convexo ${ }^{(39)}$. Em nosso trabalho, ainda que tenhamos utilizado transdutor convexo, não foi possível identificar a superfície diafragmática anterior do fígado em todos os casos.

Nossos resultados mostraram que a medida do diâmetro CCA-LHC, apesar das dificuldades técnicas já citadas, não apresentou diferença significativa no estudo de reprodutibilidade intra-observador, confirmando, portanto, sua validade, em consonância com os demais trabalhos.

\section{b) $\mathrm{CCP}-\mathrm{LHC}$}

O outro parâmetro que utilizamos para a avaliação do comprimento hepático na LHC foi o diâmetro CCP-LHC, tomando como referência a interface hepato-diafragmática posterior, que, devido à ausência da interposição de parênquima pulmonar, mostrou-se nítida em todos os casos. Assim, buscamos uma alternativa ao diâmetro CCA-LHC, com o objetivo de evitar a imprecisão da interface hepato-diafragmática anterior.

Outros métodos ultra-sonográficos relatados na literatura também propõem formas alternativas de medida do comprimento hepático na $\mathrm{LHC}^{(\mathbf{3 0 , 4 1 , 4 3 )}}$. Contudo, avaliamos que os pontos de referência estabelecidos por esses métodos ou são passíveis de imprecisão ${ }^{(30)}$ ou não são definidos com clareza $^{(43)}$, comprometendo, em qualquer dos casos, a reprodutibilidade dos méto- dos. Um dos parâmetros propostos para a avaliação das dimensões do fígado na LHC é o maior eixo entre a cúpula diafragmática e a borda ântero-inferior hepática ${ }^{(41)}$. Porém, a falta de informações quanto aos reparos anatômicos utilizados nesse trabalho faculta a escolha de pontos de referência distintos, de acordo com a estimativa de cada examinador. Em nossa investigação observamos que o maior eixo hepático na LHC é o diâmetro CCP-LHC, uma vez que a imagem do fígado, em corte longitudinal, tem conformação grosseiramente semelhante à de um triângulo retângulo, cujo maior diâmetro é a hipotenusa.

A variabilidade intra-observador da medida do diâmetro CCP-LHC não apresentou diferença significativa, confirmando sua validade.

Em relação às particularidades técnicas, podemos afirmar que a obtenção da medida do diâmetro CCP-LHC mostrou-se mais fácil de realizar que a do CCA-LHC, uma vez que seus reparos anatômicos são melhor estabelecidos.

\section{Linha axilar anterior (Figura 4)}

$\mathrm{Na}$ LAA observaram-se dificuldades técnicas relativas a: a) determinação precisa do plano de corte; b) definição dos reparos anatômicos.

Devido à curvatura do arcabouço costal coincidir com a LAA, a determinação do plano de corte nesta linha mostrou-se trabalhosa, havendo dificuldade de se obter posicionamento do transdutor no sentido ortogonal em relação ao plano da coluna. Observamos a tendência de deslocamento do transdutor no sentido lateral, produzindo cortes no eixo coronal, em vez de cortes no eixo sagital, como estabelecido pelo método. A correção do defeito de posicionamento exigiu diversas tentativas até se obter uma imagem adequada, aumentando o tempo de exame. Conseqüentemente, houve piora das condições técnicas, pois o exame prolongado contribuiu para maior inquietação da criança. A dificuldade de posicionamento do transdutor na LAA, em prejuízo da precisão do plano de corte, não foi compensada nem mesmo com a utilização do reparo anatômico intra-hepático (ramo portal direito).

Quanto à definição dos reparos anatômicos para o procedimento das medidas, 
observamos imprecisão da interface hepato-diafragmática anterior em um paciente $(3,12 \%)$, prejudicando a mensuração do diâmetro CCA-LAA. No exame de outras duas crianças $(6,25 \%)$ a medida do diâmetro CCA-LAA ficou comprometida por não se conseguir visualizar, numa mesma imagem, as extremidades superior e inferior do fígado, que excediam o limite do ângulo do feixe acústico determinado pelo transdutor convexo. Ainda que alguns autores

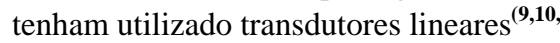
${ }^{32,38)}$ e, com isso, evitado essa limitação técnica, em nosso estudo optamos por utilizar transdutor convexo, considerando suas vantagens em relação àqueles: menor área de contato com a pele, que facilita o deslizamento e a mobilidade do transdutor e propicia melhor posicionamento entre os arcos costais; abertura do feixe na porção mais distante da fonte, oferecendo maior campo de visão nos planos profundos.

Apesar de nossos resultados terem revelado parâmetros reprodutíveis na LAA, consideramos este plano impróprio para a avaliação do tamanho do fígado em crianças, devido ao elevado grau de dificuldade técnica encontrado na obtenção destes.

\section{Análise estatística}

Quanto ao estudo da variabilidade interobservador, verificamos que não houve diferença estatisticamente significante entre as medidas obtidas nos exames 1 e 2 , indicando a reprodutibilidade de todos os parâmetros avaliados, conforme exposto na Tabela 1. Nossos achados são concordantes com os relatados por Hessel $^{(\mathbf{9})} \mathrm{em}$ seu estudo de hepatometria em crianças.

Não verificamos correlação estatisticamente significante entre as idades das crianças e as diferenças das medidas obtidas nos exames 1 e 2 . Isto sugere que o método pode ser utilizado para avaliação do tamanho do fígado na faixa etária de 0 a 6 anos. Entretanto, para os parâmetros APLME e CCP-LHC, observamos correlação negativa entre a variação das medidas e as idades das crianças, ocorrendo maiores variações nas crianças com idades menores (Tabela 2). Para justificar essas discrepâncias das medidas, aventamos a possibilidade de terem ocorrido devido ao uso de transdutor de $5 \mathrm{MHz}$ em todas as crianças examinadas. $\mathrm{O}$ uso de transdutor de fre- qüência relativamente baixa $(5 \mathrm{MHz}) \mathrm{em}$ crianças pequenas pode ter propiciado a localização dos reparos anatômicos superficiais aquém da zona focal do feixe sonoro, tornando-os imprecisos. Este efeito pode, ainda, ter sido potencializado pela distensão gasosa do estômago, comum em lactentes. Esta víscera, distendida, pode produzir um deslocamento anterior do lobo hepático esquerdo, tornando imprecisas as referências para a medida do APLME. Além disso, a grande quantidade de ar na porção gástrica fronteiriça ao fígado pode ocasionar a perda de definição da superfície hepática visceral, comprometendo a demarcação da extremidade inferior do lobo hepático direito, usada como referência para a medida do CCP-LHC. Talvez esses sejam alguns dos fatores responsáveis pelas maiores variações, ainda que estatisticamente não-significantes, nas faixas etárias mais baixas.

A análise de correlação entre os parâmetros mostrou que todos, à exceção de AP-LME e CCP-LAA, estão direta e altamente correlacionados entre si (Tabela 3 ). Portanto, para a avaliação do tamanho do fígado, qualquer desses parâmetros, exceto AP-LME e CCP-LAA, pode ser igualmente usado. Assim, é possível escolher, dentre os parâmetros, aquele(s) de mais fácil obtenção, não havendo necessidade do emprego de grande número de medidas.

\section{CONCLUSÃO}

Com base no apresentado, concluímos que o método aqui proposto é reprodutível por um mesmo observador, indicando que pode ser usado na determinação das dimensões hepáticas em crianças, na faixa de 0 a 6 anos.

Uma vez que todos os parâmetros, exceto o AP-LME, mostraram-se altamente correlacionados entre si, sugerimos a simplificação do protocolo de medidas, mantendo apenas dois parâmetros: 1) o diâmetro CC-LME, para estimativa das dimensões do lobo hepático esquerdo, tendo como reparo anatômico intra-hepático a veia hepática esquerda; e 2) o diâmetro CCP-LHC, para estimativa das dimensões do lobo hepático direito, tendo como reparo anatômico intra-hepático o ramo portal direito.
Os parâmetros CC-LME e CCP-LHC foram os de mais fácil execução, com pontos de referência precisos e altos coeficientes de correlação com as demais medidas (r variando de 0,73-0,83 e 0,73-0,86, respectivamente). Propomos que seja utilizada a medida do diâmetro CCP-LHC em vez do usual CCA-LHC, não só pelas vantagens técnicas, mas também por incluir uma superfície maior do fígado. Desse modo, pode vir a detectar aumento do órgão que, eventualmente, não se expresse com aumento do seu comprimento ("liver span").

Em virtude de termos introduzido novo parâmetro para avaliação das dimensões do fígado (CCP-LHC), torna-se necessário, futuramente, estabelecer uma tabela de valores normais, tomando-se como base a nova referência. A inclusão de outros examinadores permitirá estudar a reprodutibilidade interobservador dos parâmetros estabelecidos neste método.

\section{REFERÊNCIAS}

1. Naftalis J, Leevy CM. Clinical estimation of liver size. Am J Dig Dis 1963;8:236-43.

2. Walk L. Assessment of liver size. Digestion 1968; 1:289-95.

3. Rosenfield AT, Schneider PB. Rapid evaluation of hepatic size on radioisotope scan. J Nucl Med 1974; 15:237-40.

4. Younoszai MK, Mueller S. Clinical assessment of liver size in normal children. Clin Pediatr (Phila) 1975;14:378-80.

5. Carpentieri U, Gustavson LP, Leach TM, Bunce H III. Liver size in normal infants and children. South Med J 1977;70:1096-7.

6. Lawson EE, Grand RJ, Neff RK, Cohen LF. Clinical estimation of liver span in infants and children. Am J Dis Child 1978;132:474-6.

7. Reiff MI, Osborn LM. Clinical estimation of liver size in newborn infants. Pediatrics 1983;71:46-8

8. Walk L. Liver size in children determined with quantitative methods. Radiologe 1985;25:221-3.

9. Hessel G. Hepatometria na infância - comparação entre o método clínico e ultra-sonográfico. (Dissertação de Mestrado). Campinas, SP: Faculdade de Ciências Médicas da Universidade Estadual de Campinas, 1991.

10. Chen CM, Wang JJ. Clinical and sonographic assessment of liver size in normal Chinese neonates. Acta Paediatr 1993;82:345-7.

11. Walker WA, Mathis RK. Hepatomegaly. An approach to differential diagnosis. Pediatr Clin North Am 1975;22:929-42.

12. Castell DO, O'Brien KD, Muench H, Chalmers TC. Estimation of liver size by percussion in normal individuals. Ann Intern Med 1969;70:1183-9.

13. Deligeorgis D, Yannakos D, Panayotou P, Doxiadis $\mathrm{S}$. The normal borders of the liver in infancy and childhood. Clinical and X-ray study. Arch Dis Child 1970;45:702-4.

14. Sullivan S, Krasner N, Williams R. The clinical 
estimation of liver size: a comparison of techniques and an analysis of the source of error. Br Med J 1976;2:1042-3.

15. Bricks LF, Kobinger MEBA, Rañna W. Hepatoesplenomegalia. In: Marcondes E. Pediatria básica. $8^{\text {a }}$ ed. São Paulo, SP: Sarvier, 1991:193-7.

16. Weisman LE, Cagle N, Mathis R, Merenstein GB. Clinical estimation of liver size in the normal neonate. Clin Pediatr (Phila) 1982;21:596-8.

17. Walk L. Roentgenologic determination of the liver volume. Acta Radiol 1961;55:49-56.

18. Walk L. Quantitative method to determine the liver size. Radiologe 1978;18:354-5.

19. Walk L. Normal liver size as determined with quantitative methods. Radiologe 1982;22:188-9.

20. Peternel WW, Schaefer JW, Schiff L. Clinical evaluation of liver size and hepatic scintiscan. Am J Dig Dis 1966;11:346-50.

21. Markisz JA, Treves ST, Davis RT. Normal hepatic and splenic size in children: scintigraphic determination. Pediatr Radiol 1987;17:273-6.

22. Jones KR, Robinson PJ. Organ volume determination by CT scanning: reduction of respiration induced errors by feed back monitoring. J Comput Assist Tomogr 1986;10:167-71.

23. Kardel T, Holm HH, Rasmussen SN, Mortensen T. Ultrasonic determination of liver and spleen volumes. Scand J Clin Lab Invest 1971;27:123-8.

24. Holder LE, Strife J, Padikal TN, Perkins PJ, Kereiakes JG. Liver size determination in pediatrics using sonographic and scintigraphic techniques. Radiology $1975 ; 117: 349-53$.
25. Carr D, Duncan JG. Liver volume determination by ultrasound: a feasibility study. Br J Radiol 1976;49: 776-8.

26. Holmes JH, Sundgren C, Ikle D, Finch J. A simple ultrasonic method for evaluating liver size. J Clin Ultrasound 1977;5:89-91.

27. Wladimiroff JW, Sekeris A. Ultrasonic assessment of liver size in the newborn. J Clin Ultrasound 1977;5:316-20.

28. Rasmussen SN. Liver volume determination by ultrasonic scanning. Dan Med Bull 1978;25:1-46.

29. Sapira JD, Williamson DL. How big is the normal liver? Arch Intern Med 1979;139:971-3.

30. Gosink BB, Leymaster CE. Ultrasonic determination of hepatomegaly. J Clin Ultrasound 1981;9: 37-44.

31. Rylance GW, Moreland TA, Cowan MD, Clark DC. Liver volume estimation using ultrasound scanning. Arch Dis Child 1982;57:283-6.

32. Dittrich M, Milde S, Dinkel E, Baumann W, Weitzel D. Sonographic biometry of liver and spleen size in childhood. Pediatr Radiol 1983;13:206-11.

33. Niederau C, Sonnenberg A, Müller JE, Erckenbrecht JF, Scholten T, Fritsch WP. Sonographic measurements of the normal liver, spleen, pancreas, and portal vein. Radiology 1983;149:537-40.

34. Skrainka B, Stahlhut J, Fulbeck CL, Knight F, Holmes RA, Butt JH. Measuring liver span: bedside examination versus ultrasound and scintiscan. J Clin Gastroenterol 1986;8(3 Pt 1):267-70.

35. Baddeley H, Benson M, Liefman G, et al. Measurement of liver volume using water delay ultrasonog- raphy. Diagn Imag Clin Med 1986;55:330-6.

36. Phuapradit P, Assadamongkol K, Udompanich O, Varavithya W. Liver size and serum alkaline phosphatase in normal preschool Thai children. J Med Assoc Thai 1986;69 Suppl 2:69-76.

37. Assadamongkol K, Phuapradit P, Udompanich O, Varavithya W. Liver size and serum alkaline phosphatase in normal Thai school-aged children. J Med Assoc Thai 1989;72 Suppl 1:88-93.

38. Friis H, Ndhlovu P, Mduluza T, et al. Ultrasonographic organometry: liver and spleen dimensions among children in Zimbabwe. Trop Med Int Health 1996;1:183-90.

39. Konus ÖL, Özdemir A, Akkaya A, Erbas G, Celik $\mathrm{H}$, Isik S. Normal liver, spleen, and kidney dimensions in neonates, infants, and children: evaluation with sonography. AJR 1998;171:1693-8.

40. Jungthirapanich J, Kaewtubtim J, Poovorawan Y. A new reference line for measuring the liver size in healthy newborns. J Med Assoc Thai 1998;81:93843.

41. Haddad-Zebouni S, Hindy R, Slaba S, et al. Evaluation échographique de la taille des reins, du foie et de la rate chez l'enfant. Arch Pédiatr 1999;6 1266-70.

42. Naylor CD, McCormack DG, Sullivan SN. The midclavicular line: a wandering landmark. CMAJ 1987;136:48-50.

43. Cosgrove D. Liver anatomy. In: Meire HB, Cosgrove DO, Dewbury KC, Farrant P, eds. Clinical ultrasound - a comprehensive text. 2nd ed. London, UK: Churchill Livingstone, 2001:165-81. 